\title{
The Possible Role of SCO3388, a tmrB-like Gene of Streptomyces coelicolor, in Germination and Stress Survival of Spores
}

\author{
So-Yeon Kwon · Hyung-Jin Kwon*
}

Received: 14 February 2013 / Accepted: 22 May 2013 / Published Online: 30 September 2013

(C) The Korean Society for Applied Biological Chemistry 2013

\begin{abstract}
The SCO3388 gene from Streptomyces coelicolor is homologous to $\operatorname{tmr} B$, the tunicamycin resistance gene of Bacillus subtilis. The SCO3388-inactivation strain (SY-tbl-1) was generated by replacing SCO3388 with thiostrepton resistance gene. Spores of $S$. coelicolor derivatives were prepared on mannitol-soy flour (MS) agar on which SY-tbl-1 displayed no significant defect in growth and development. When plated on R4 agar, spores of SYtbl-1 displayed retardation in growth and sporulation, whereas its mycelium gave rise to normal growth. Thus, SCO3388 is suggested to be involved in the dormant spore germination. Expression of $S C O 3388$ under the ermE1 promoter restored but only partially the ability to sporulate in SY-tbl-1. Neither SY-tbl1 nor SY-tbl-1/ermE1p-SCO3388 showed a difference in tunicamycin resistance to the wild type whereas, interestingly, the introduction of ermE1p-SCO3388 dramatically enhanced spore survival to heat and detergent treatments, suggesting that SCO3388 might play a role in the maintenance of spore cell wall integrity.
\end{abstract}

Keywords SCO3388 gene $\cdot$ spore germination - Streptomyces coelicolor $\cdot$ stress survival of spores $\cdot \operatorname{tmr} B$-like

\section{Introduction}

Tunicamycin is a mixture of nucleoside antibiotics produced from some Streptomyces strains (Takatsuki et al., 1971; Kenig and Reading, 1979). It targets peptidoglycan biosynthesis at the step of UDP- $N$-acetyl-D-hexosamine:polyprenyl-phosphate $N$-acetylhexosamine-1-phosphate translocase (Billot-Klein et al., 1997). Tunicamycin is also well known for its inhibitory activity on protein $N$ -

S.-Y. Kwon · H.-J. Kwon

Department of Biological Science, Division of Bioscience and Bioinformatics, Myongji University, Yongin-si, Gyeonggi-do 449-728, Republic of Korea

*Corresponding author (H.-J. Kwon: hjink@mju.ac.kr) glycosylation and is widely used as an endoplasmic reticulum stress-inducer in eukaryotic cell biology. As an antibiotic, tunicamycin was found to be most effective against Bacillus subtilis (Takatsuki et al., 1971). A tunicamycin-resistant mutant of $B$. subtilis harbored multiple copies of $t m r B$, which was thus assigned as tunicamycin resistance gene (Hashiguchi et al., 1986). It was later proposed that TmrB protein binds tunicamycin and is localized to cytoplasmic membrane (Noda et al., 1992; 1995). However, B. subtilis is sensitive to tunicamycin although it harbors a copy of $t m r B$ on its chromosome. In addition, the tunicamycin biosynthetic gene cluster of Streptomyces spp. has no tmrB homologue (Wyszynski et al., 2010; Karki et al., 2011). Thus, TmrB may be involved in peptidoglycan biogenesis, which is a known target of tunicamycin. High levels of TmrB may confer tunicamycin resistance by potentiating cell wall (CW) metabolism and by enhancing $\mathrm{CW}$ integrity.

Homologues of $\operatorname{tmr} B$, annotated as putative tunicamycin resistance genes or $\operatorname{tm} r B$-like genes, can be found in genome sequences of many Gram-positive bacteria but this annotation is only given because TmrB-like proteins share no significant homology with any other proteins whose physiological functions are defined. As aforementioned, the role of $\mathrm{TmrB}$ in the physiology of B. subtilis, other than in tunicamycin resistance, is unknown. It is thus necessary to examine whether the $\operatorname{tmr} B$ homologues are related to tunicamycin resistance or have other roles in the cellular physiology of Gram-positive bacteria.

Streptomyces undergo morphological development, and each developmental stage adopts a distinctive cell type: substrate mycelium, aerial mycelium, and spore. This feature makes Streptomyces a model system in studying the regulatory network controlling bacterial cell differentiation. The SCO3388 protein of S. coelicolor displays $38.9 \%$ identity to $\mathrm{TmrB}$ with 203 -amino acid overlap and is annotated as a TmrB-like protein (Supplementary data Fig. 1). SCO3388 is downstream of genes encoding a two-component system (SCO3389 and SCO3390). Transcription of SCO3388 is likely under the control of this twocomponent system. Among 29 analyzed genome sequences of 
Streptomyces spp., 9 have homologues of SCO3388 with at least $64 \%$ identity in the deduced amino acid sequences. In this study, SCO3388 was inactivated through gene-replacement and the phenotype of the resulting mutant was examined in terms of morphological differentiation, tunicamycin resistance, and stress survival of spores. Our study demonstrates that SCO3388 is involved in germination and stress survival of spores but not in tunicamycin resistance.

\section{Materials and Methods}

Strains, culture conditions, and genetic procedure. Streptomyces coelicolor M145 was used in this study. S. coelicolor strains were grown at $28^{\circ} \mathrm{C}$ on $\mathrm{R} 4$ agar, which contains $10 \mathrm{~g}$ of glucose, $1 \mathrm{~g}$ of yeast extract, $0.1 \mathrm{~g}$ of casamino acids, $3 \mathrm{~g}$ of L-proline, $10 \mathrm{~g}$ of $\mathrm{MgCl}_{2} \cdot 6 \mathrm{H}_{2} \mathrm{O}, 4 \mathrm{~g}$ of $\mathrm{CaCl}_{2} \cdot 2 \mathrm{H}_{2} \mathrm{O}, 0.2 \mathrm{~g}$ of $\mathrm{K}_{2} \mathrm{SO}_{4}, 5.6 \mathrm{~g}$ of TES ( $N$-tris [hydroxymethyl] methyl-2-aminoethanesulfonic acid), $1 \mathrm{~mL}$ of trace element solution and $20 \mathrm{~g}$ of agar per liter $(\mathrm{pH} 7.2)$ or MS (mannitol-soy flour) agar, which contains $20 \mathrm{~g}$ of mannitol, $20 \mathrm{~g}$ of soya flour, and $20 \mathrm{~g}$ of agar per liter. For liquid culture, tryptic soy broth (TSB) medium was used. TSB contains $17 \mathrm{~g}$ of casein, $3 \mathrm{~g}$ of soybean, $2.5 \mathrm{~g}$ of D-dextrose, $5 \mathrm{~g}$ of $\mathrm{NaCl}$, and 2.5 $\mathrm{g}$ of $\mathrm{K}_{2} \mathrm{HPO}_{4}$ per liter ( $\mathrm{pH}$ 7.3). S. coelicolor and its derivatives were cultivated in TSB medium with $0.5 \%$ glycine and $5 \mathrm{mM}$ $\mathrm{MgCl}_{2} \cdot 6 \mathrm{H}_{2} \mathrm{O}$ for total DNA isolation. Total DNA was isolated by using bacterial genomic DNA isolation kit (Core-one ${ }^{\mathrm{TM}}$, CoreBio System, Korea). For spore collection, TSB culture was spread on MS agar plates and incubated at $28^{\circ} \mathrm{C}$. After 5 days, spores were harvested with moistening sterile cotton soaked in sterile water. Five milliliter of sterile water was applied per one plate, wiping the surface of plates. Each stock was stored in $25 \%$ aqueous glycerol at $-70^{\circ} \mathrm{C}$. Escherichia coli $\mathrm{DH} 5 \alpha$ strain was used for routine cloning and was grown at $37^{\circ} \mathrm{C}$ in Luria-Bertani medium. Ampicillin, apramycin or thiostrepton was added when necessary at the final concentration of $50 \mu \mathrm{g} / \mathrm{mL}$ except that thiostrepton was applied at $5 \mu \mathrm{g} / \mathrm{mL}$ in the liquid culture. Intergeneric conjugal transfer was conducted to introduce DNA into $S$. coelicolor by using E. coli ET12567/pUZ8002 as the donor strain (Paget et al., 1999). Germinated spore was used for recipient cell and conjugal transfer was performed on AS-1 agar as previously described (Bierman et al., 1992).

Inactivation and expression of $\mathbf{S C O 3 3 8 8}$ in $\boldsymbol{S}$. coelicolor. The SCO3388 inactivation plasmid pSY1101 was constructed by inserting thiostrepton resistance gene (tsr) between DNA fragments flanking SCO3388 (nt 126,887 to 127,528), which were prepared by polymerase chain reaction (PCR) amplification from chromosomal DNA. The nucleotide numbers (nt) in this study are based on the sequence deposited in GenBank accession number AL939116. The oligonucleotide primers are listed in Table 1. The $2.0-\mathrm{kb}$ upstream region (nt 127,478 to 129,540 ) was amplified by polymerase chain reaction (PCR) with the primer pair of $\mathrm{d} 3388$ 1 and $\mathrm{d} 3388-2$. The $2.0-\mathrm{kb}$ downstream region (nt 124,801 to $126,960)$ was amplified by PCR with the primer pair of $\mathrm{d} 3388-3$
Table 1 Primers used in this study

\begin{tabular}{|c|c|}
\hline Pimer & Sequence* \\
\hline d3388-1 & 5'-ATTTCTAGAGGTTACGTGCAGCGCCTCTA-3' \\
\hline $\mathrm{d} 3388-2$ & 5'-ATTAAGCTTGCGGGCAGCGACTGGATGGA-3' \\
\hline d3388-3 & 5'-ATTGAATTCCGAACAACGAGGGCGCCCTG-3' \\
\hline d $3388-4$ & 5'-ATTACTAGTTCTCGTTCTACAAGTCCCTC-3'. \\
\hline $3388 f$ & 5'-ATTTCGTCGGTAGGGTCTGCGCA-3' \\
\hline $3388 \mathrm{r}$ & 5'-ATTACTTCCTGGAGGGGATACTC-3' \\
\hline $3388 \mathrm{ex}-\mathrm{f}$ & 5'-ATTAAGCTTATGTCCAACGGCGAGATCGC-3' \\
\hline 3388 ex-r & 5'-ATTTCTAGATACCTGGCCCGCAGCGCG-3' \\
\hline
\end{tabular}

$* 5$ ' unmatched sequences and engineered restriction sites are underlined

and $\mathrm{d} 3388-4$. The upstream fragment was ligated into the $\mathrm{Xba \textrm {I } /}$ HindIII sites of pHJK-3-14C (Kim et al., 2008). To the resulting plasmid, the downstream fragment was ligated into the EcoRI/ SpeI sites. The insert of the resulting plasmid was rescued as $\mathrm{Xba \textrm {I } /}$ SpeI fragment and ligated into the $X b a \mathrm{I}$ site of pOJ260 (Bierman et al., 1992). The resulting plasmid pSY1101 was introduced into $S$. coelicolor M145 by intergeneric conjugation. The double crossover mutant of SY-tbl-1 (ASCO3388::tsr) was selected by thiostrepton-resistant and apramycin-sensitive phenotype. Inactivation of SCO3388 in SY-tbl-1 was confirmed by PCR with the primer pairs of 3388f and 3388r (nt 126,819 to 127,555).

For the expression construct, SCO3388 was amplified by PCR with the primer pairs of 3388ex-f and 3388ex-r (nt 126,641 to 127,750 ). The PCR product was ligated into the $X b a \mathrm{I} / H$ HindIII sites of the pLitmus 28 to construct pSCOTBL2. The SpeI/XbaI fragment of pSCOTBL2 was then ligated into XbaI site of pSET152 (Bierman et al., 1992) harboring the ermE promoter $\left(e r m E^{*} \mathrm{p}\right)$. Restriction digestion analysis was use to select the clone harbor SCO3388 at the transcription direction of emrE1p to generate pSCO3388. A 270-bp emrE promoter region was amplified by PCR with engineered restriction sites of $X b a \mathrm{I}$ and HindIII at the transcriptional directions of ermE1p and ermE2p, respectively (Bibb et al., 1985). This divergent promoter fragment was cloned in XbaI and HindIII sites of pSET152.

Sodium dodecylsulfate (SDS) treatment and heat shock of spore. The concentrations of the spore suspensions were adjusted to be $5.0 \times 10^{8} / \mathrm{mL}$. The spore suspensions were diluted by $10^{5}$-fold with sterile water and exposed to $5 \%$ SDS for $5 \mathrm{~h}$ at ambient temperature. For the heat treatment, the diluted spores were incubated at $60^{\circ} \mathrm{C}$ for $10 \mathrm{~min}$. The treated spores were spread on MS agar plates and were incubated at $28^{\circ} \mathrm{C}$ for 4 days to count colony forming unit (CFU). Survival rates were calculated by dividing the number of colonies on plates by the number of colonies generated from the spore sample without any treatment. Scanning and transmission electron microscope (SEM and TEM) examination. The hyphase of S. lividans WT and SY-tbl1 strain were examined under SEM. Blocks of the R4 agar cultures, which were incubated at $28^{\circ} \mathrm{C}$ for 7 days, were fixed with $1 \%$ osmium tetroxide for $12 \mathrm{~h}$ and then dehydrated by freeze- 
drying. Each specimen was sputter coated with platinum-gold and examined under a Hitachi S4000 SEM.

TEM was used to observe spores of $S$. lividans derivatives. Spore pellets were fixed at $4^{\circ} \mathrm{C}$ for $4 \mathrm{~h}$ in $2 \%$ paraformaldehyde and $2 \%$ glutaraldehyde in $50 \mathrm{mM}$ sodium cacodylate buffer $(\mathrm{pH}$ 7.2) and were then rinsed three times (at $4^{\circ} \mathrm{C}$ for $10 \mathrm{~min}$ ) with the same buffer. The pellets were post-fixed at $4^{\circ} \mathrm{C}$ for $2 \mathrm{~h}$ with $1 \%$ osmium tetroxide $\mathrm{OsO}_{4}$ in the same buffer and washed two times with distilled water. An en bloc staining was done at $4^{\circ} \mathrm{C}$ for 28 min with $0.5 \%$ uranyl acetate. Specimens were dehydrated through a graded ethanol solution, transferred to propylene oxide, embedded in Spur's resin and polymerized at $70^{\circ} \mathrm{C}$ for $24 \mathrm{~h}$. Ultrathin sections were obtained with MT-X ultramicrotome (RMC, USA). To improve stain, the sections were stained with $2 \%$ uranyl acetate for $7 \mathrm{~min}$ and Reynolds' lead citrate for $7 \mathrm{~min}$. The sections were examined using LIBRA 120 TEM (Carl Zeiss, Germany).

\section{Results}

Inactivation of $\mathrm{SCO} 3388$ resulted in defective spore development. The SCO3388-inactivation strain (SY-tbl-1) was generated by replacing $S C O 3388$ with a thiostrepton resistance gene (tsr) (Fig. 1A). The SCO3388-inactivation construct pSY1101 was introduced into $S$. coelicolor by intergeneric conjugation; exconjugants were selected with thiostrepton resistance. pSY1101 is a derivative of pOJ260, which is incapable of replicating in Streptomyces (Bierman et al., 1992). The thiostrepton-resistant exconjugants were then tested for aparmycin resistance and a double crossover mutant of SY-tbl-1 with the thiostreptonresistant/aparmycin-sensitive phenotype was selected. Exconjugants displaying the resistance to both thiostrepton and aparmycin are single crossover mutants. PCR amplification was used to confirm the genotype of the mutants (Fig. 1B). PCR of S. coelicolor M145 wild-type strain (WT) resulted in a $0.74-\mathrm{kb}$ band (lane 1), which was replaced with a 1.2-kb fragment in SY-tbl-1 (lane 2). Both the $0.74-\mathrm{kb}$ and $1.2-\mathrm{kb}$ fragments were found in the single crossover mutant (lane 3). This result substantiated that SCO3388 was successfully knocked out in SY-tbl-1. Southern hybridization analysis further verified that SCO3388 was eliminated in SY-tbl1 (Supplementary data Fig. 2). Morphological comparison of mycelia, obtained from TSB liquid cultures of WT and SY-tbl-1, indicated that there was no significant difference when spread on R4 agar plates (Fig. 1C; -m). Spores of SY-tbl-1 were obtained on MS agar. When plated on R4 medium, these spores displayed a significant defect in growth (Fig. 1C; -s). SEM examination showed that SY-tbl-1 produced aerial hyphae thinner than WT (Supplementary data Fig. 3). It was found that SY-tbl-1displayed a significantly lower level of growth than WT on R4 agar while its growth level was measured higher than WT on MS agar (Fig. 1D).

Gene complementation partially restored sporulation to SYtbl-1. SCO3388 was cloned in pSET152 under the control of
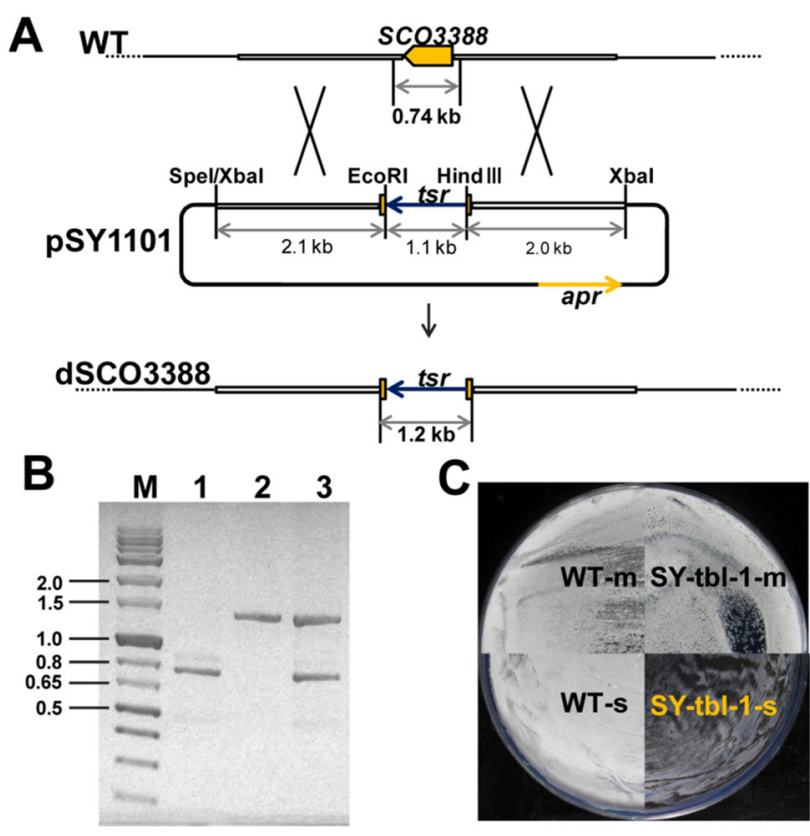

D 1.R4 2.MS

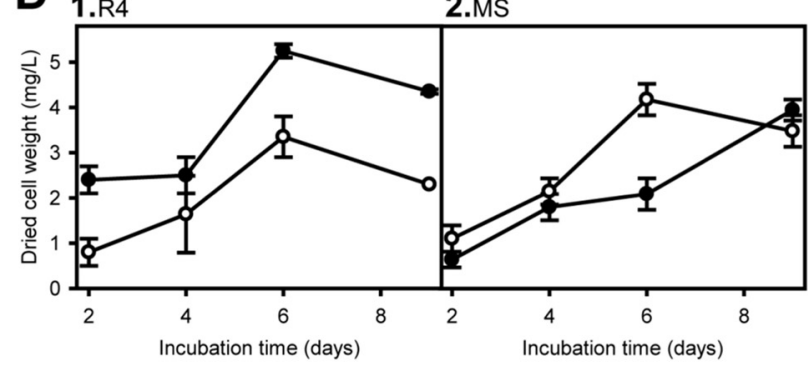

Fig. 1 Gene inactivation of SCO3388 by double crossover-mediated gene replacement and the visual examination of SY-tbl-1. (A) Schematic representation of the homologous recombination that generates SY-tbl-1 and the restriction map of the gene-inactivation plasmid pSY1101 showing the predicted PCR product sizes with primer pair 3388f-3388r from WT and SY-tbl-1 strains. (B) Electrophoretic analysis of the PCR performed with the chromosomes of WT (1), dSCO3388 (2), and a single crossover mutant (3). Lane $\mathrm{M}$ indicates the DNA molecular weight marker with the size indication bars at the relevant fragments. (C) Visual examination of growth of WT and SY-tbl-1. Mycelia (-m), which were obtained from TSB liquid culture, or Spores (-s) were overlaid on R4 agar and incubated at $28^{\circ} \mathrm{C}$ for 4 days. (D) Growth of WT (filled circles) and SY-tbl-1 (open circles) on R4 agar (1) and MS agar (2). The agar media were covered with autoclaved cellophane disks and then overlaid with $10^{7}$ of spores. The cultures were incubated at $28^{\circ} \mathrm{C}$ and the mycelia were harvested with distilled water at the incubation time indicated. The mycelia were collected by centrifugation and dried at $60^{\circ} \mathrm{C}$ for 2 days before the dried cell weights were measured.

emrE1p. The resulting construct named pSCO3388 was introduced into SY-tbl-1. Morphology examination was done on either R4 or MS agar by applying approximately 500 spores on each plate. Introduction of pSCO3388 into SY-tbl-1 partially restored the ability to sporulate on R4 agar (Fig. 2A). The partial complementation with pSCO3388 may be due to the usage of an unnatural promoter (emrE1p) in SCO3388 expression. Transformation of SY-tbl-1 with $S C O 3388$ without emrElp failed to restore the sporulating 


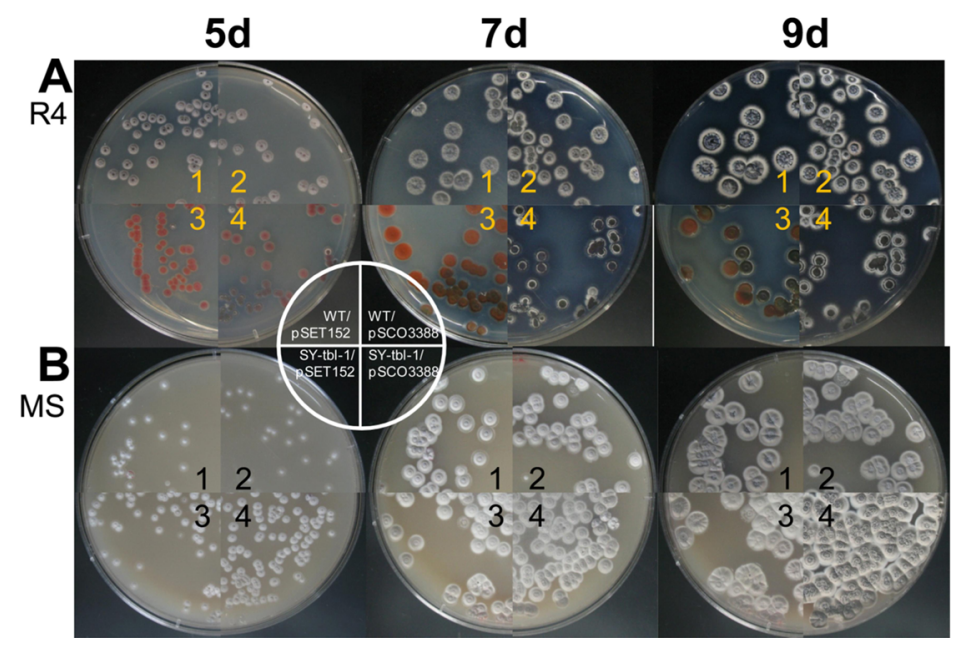

Fig. 2 Development of SY-tbl-1 and the gene complementation strain on R4 and MS agar. Time course (5 days, 7 days, and 9 days after culture initiation) examination of WT/pSET152 (1), WT/pSCO3388 (2), SY-tbl-1/pSET152 (3) and SY-tbl-1/pSCO3388 (4) on R4 agar (A) or MS agar (B). Approximately 500 spores were applied on each plate and incubated at $28^{\circ} \mathrm{C}$.

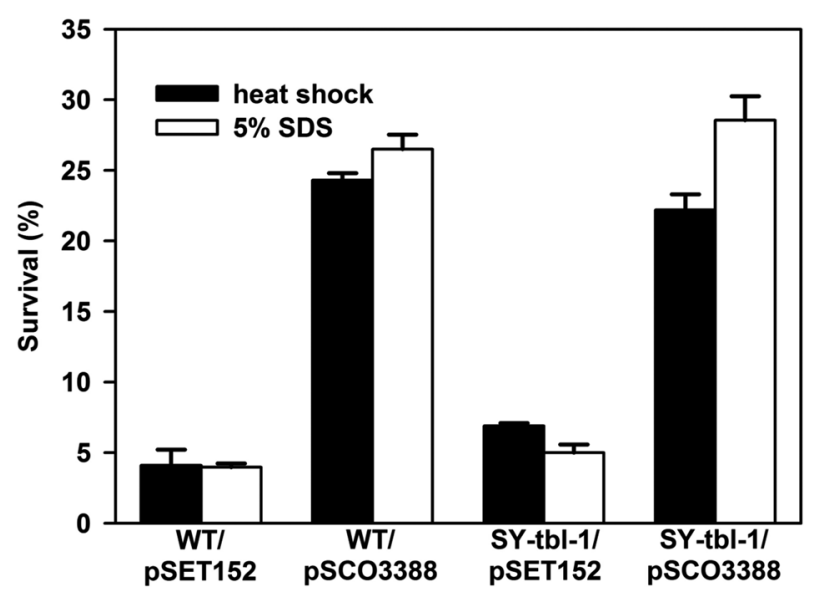

Fig. 3 Stress survival test. Experiments were carried out three times; standard errors are provided as error bars.

phenotype (data not shown). In MS agar, SY-tbl-1 showed no defect in sporulation, displaying accelerated growth and a delay in spore pigmentation (Figs 1D and 2B).

Tunicamycin resistance was not affected by SCO3388. A preliminary test of tunicamycin resistance indicated that $S$. coelicolor had substantial resistance to tunicamycin and there was no inhibition zone in either WT or SY-tbl-1 when tunicamycin was tested at amounts up to $20 \mu \mathrm{g}$ zone. Application of $200 \mu \mathrm{g}$ tunicamycin gave rise to small inhibitions in both strains. To compare the tunicamycin resistance of WT/pSET152, SY-tbl-1/ pSET152, and SY-tbl-1/pSCO3388, a paper disk $(\square, 1 \mathrm{~cm})$ containing $1.2 \mathrm{mg}$ of tunicamycin (Sigma, USA) was applied on a plate which was overlaid with approximately $3 \times 10^{7}$ spores. There was no significant difference in the inhibition zone between strains (Fig. S4), indicating that SCO3388 is not a determining factor of tunicamycin resistance in $S$. coelicolor.
Expression of $\mathbf{S C O} 3388$ enhanced stress survival. It was previously reported that inactivation of cell wall hydrolases resulted in heat sensitivity, defects in spore formation, delay in spore germination and vegetative growth suggesting a key role of these enzymes in cell wall metabolism throughout $S$. coelicolor life cycle (Haiser et al., 2009). To assess the possibility that SCO3388 was also involved in spore cell wall metabolism, stress survival was tested with spores of the recombinant strains. Following heat shock and 5\% SDS treatment, SY-tbl-1/ pSCO3388 displayed a dramatic increase in spore survival (Fig. 3), suggesting that the expression of SCO3388 enhanced spore cell wall integrity. WT/pSCO3388 also displayed a dramatic increase in the heat shock-resistance up to the level observed in SY-tbl-1/pSCO3388 (Fig. 3). Spore survival tests with $2 \%$ and $10 \%$ SDS showed patterns similar to those seen with 5\% SDS (data not shown). These observations suggest that SCO3388 is playing a role in spore cell wall metabolism and its integrity. This assumption led us to examine spore cell wall structure of SY-tbl1with TEM (Fig. 4). The cell wall of SY-tbl-1/pSET152 appeared darker (denser) and thinner than that of the WT/pSET152 and SYtbl-1/pSCO3388 but the physiological significance of this apparent modification of the cell wall structure is still unknown.

\section{Discussion}

Multiple alignment analysis of several TmrB homologues indicates that there are some strictly conserved residues, the most conserved of which are the $N$-terminal regions (Supplementary data Fig. 1). The $N$-terminal region contains the characteristic Walker A ATP-binding motif (GXXGXGKT) (Walker et al., 1982), which is not found in SCO3388. The possibility that SCO3388 is a variant TmrB-like protein that lost its ATP-binding activity cannot be excluded, but it is unclear whether TmrB-like 


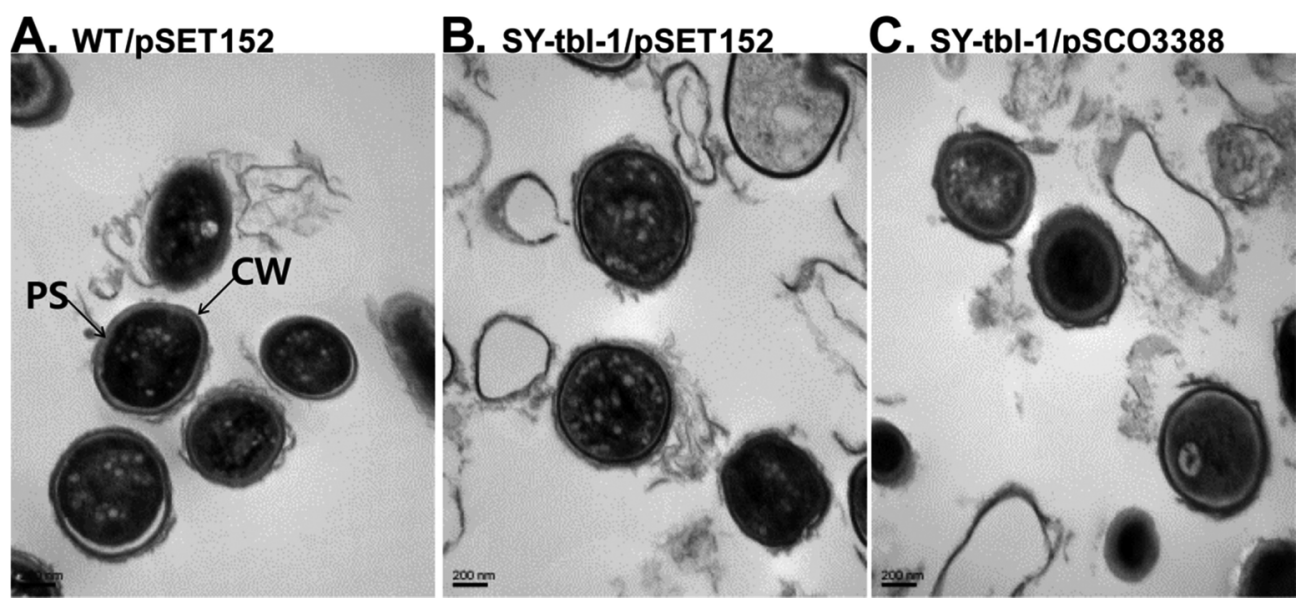

Fig. 4 Transmission electron microscopy. Microscopic inspection of WT/pSET152 (A), SY-tbl-1/pSET152 (B), and SY-tbl-1/pSCO3388 (C) at 60,000× magnification. Cell wall (CW) and periplasmic space (PS) are indicated by arrows.

proteins, including TmrB of B. subtilis, employ ATP-binding to perform their biological roles.

The $\Delta S C O 3388:: t s r$ mutant (SY-tbl-1) displayed defects in growth and sporulation on R4 agar (Figs. 1 and 2), which was partially complemented when ermE1p-SCO3388 (pSCO3388) was introduced into this mutant (Fig. 2A). Significantly, this result is the first demonstration that a TmrB-like protein has a role in bacterial development. Morphological differentiation of SY-tbl-1 was normal in MS agar (Fig 2B), suggesting that SY-tbl-1 is a conditional mutant.

Germination of dormant spores demands CW remodeling, involving CW hydrolysis (Haiser et al., 2009). Development of spores also requires $\mathrm{CW}$ assembly in addition to component synthesis, as shown in mre mutants (Mazza et al., 2006). The CW of $r p f A$ (CW hydrolase gene) mutant is thinner and denser than WT (Haiser et al., 2009), while the mre mutants display severely deformed spores (Mazza et al., 2006). Spores of SY-tbl-1 possessed a dense (dark) $\mathrm{CW}$ with a limited PS layer compared to WT, resembling the rpfA mutant (Fig. 4). This observation may indicate that the loss of SCO3388 does not affect CW assembly itself but rather $\mathrm{CW}$ remodeling in the spore germination stage. The true nature of the layer underlying the $\mathrm{CW}$ layer was unclear, but this layer was assumed to be PS in the present study, according to the electron microscopic dissection study of $B$. subtilis CW (Matias and Beveridge, 2008).

A mutation in either $\mathrm{CW}$ hydrolase or mre gene (spore $\mathrm{CW}$ assembly) makes the cognate mutant sensitive to stress conditions, such as heat shock (Mazza et al., 2006; Haiser et al., 2009), but SY-tbl-1 displayed no decrease in stress survival (Fig. 3). Stress survival of SY-tbl-1 was moderately higher than WT (Fig. 3), whereas its spore was defective in morphological development (Figs. 1 and 2). Introduction of ermE1p-SCO3388 dramatically enhanced stress survival of S. coelicolor (Fig. 3), while the expression strain (SY-tbl-1/pSCO3388) displayed no significant difference to WT in the spore CW appearance under TEM (Fig. 4). Thus, stress survival was not related to the appearance of the spore $\mathrm{CW}$ structure under TEM. We therefore propose that SCO3388 is a novel factor in spore CW remodeling and morphological differentiation in S. coelicolor and that TmrB-like proteins have a role in $\mathrm{CW}$ metabolism in Gram-positive bacteria.

Acknowledgments This work was supported by the Korea Research Foundation Grant funded by the Korean Government (MOEHRD, Basic Research Promotion Fund) (2011-0021169) and a grant from the Next-Generation BioGreen 21 Program (PJ009643), Rural Development Administration, Republic of Korea.

\section{References}

Bibb MJ, Janssen GR, and Ward JM (1985) Cloning and analysis of the promoter region of the erythromycin resistance gene (ermE) of Streptomyces erythraeus. Gene 38, 215-26.

Bierman M, Logan RK, Brien O, Seno ET, Rao RN, and Schoner BE (1992) Plasmid cloning vectors for the conjugal transfer of DNA from Escherichia coli to Streptomyces spp. Gene 116, 43-9.

Billot-Klein D, Shlaes D, Bryant D, Bell D, Legrand R, Gutmann L et al. (1997) Presence of UDP- $N$-acetylmuramyl-hexapeptides and -heptapeptides in Enterococci and Staphylococci after treatment with ramoplanin, tunicamycin, or vancomycin. J Bacteriol 179, 4684-8.

Haiser HJ, Yousef MR, and Elliot MA (2009) Cell wall hydrolases affect germination, vegetative growth, and sporulation in Streptomyces coelicolor. J Bacteriol 191, 6501-12.

Hashiguchi K, Tanimoto A, Nomura S, Yamane K, Yoda K, Harada S et al. (1986) Amplification of the $a m y E$-tmrB region on the chromosome in tunicamycin-resistant cells of Bacillus subtilis. Mol Gen Genet 204, 3643.

Karki S, Kwon SY, and Kwon HJ (2011) Cloning of tunicamycin biosynthetic gene cluster from Streptomyces chartreusis NRRL 3882. J Korean Soc Appl Biol Chem 54, 136-40.

Kenig M and Reading C (1979) Holomycin and an antibiotic (MM 19290) related to tunicamycin, metabolites of Streptomyces clavuligerus. $J$ Antibiot 32, 549-54.

Kim JY, Suh JW, Kang SH, Phan TH, Park SH, and Kwon HJ (2008) Gene inactivation study of gntE reveals its role in the first step of pseudotrisaccharide modifications in gentamicin biosynthesis. Biochem 
Biophys Res Commun 372, 730-4.

Matias VR and Beveridge TJ (2008) Lipoteichoic acid is a major component of the Bacillus subtilis periplasm. $J$ Bacteriol 190, 7414-8.

Mazza P, Noens EE, Schirner K, Grantcharova N, Mommaas AM, Koerten HK et al. (2006) MreB of Streptomyces coelicolor is not essential for vegetative growth but is required for the integrity of aerial hyphae and spores. Mol Microbiol 60, 838-52.

Noda Y, Yoda K, Takatsuki A, and Yamasaki M (1992) TmrB protein, responsible for tunicamycin resistance of Bacillus subtilis, is a novel ATP-binding membrane protein. $J$ Bacteriol 174, 4302-7.

Noda Y, Takatsuki A, Yoda K, and Yamasaki M (1995) TmrB protein, which confers resistance to tunicamycin on Bacillus subtilis, binds tunicamycin. Biosci Biotechnol Biochem 59, 321-2.
Paget MS, Chamberlin L, Atrih A, Foster SJ, and Buttner MJ (1999) Evidence that the extracytoplasmic function sigma factor $\mathrm{E}$ is required for normal cell wall structure in Streptomyces coelicolor A3(2). J Bacteriol 181, 204-11.

Takatsuki A, Arima K, and Tamura G (1971) Tunicamycin, a new antibiotic. I. Isolation and characterization of tunicamycin. $J$ Antibiot 24, 215-23.

Walker JE, Saraste M, Runswick MJ, and Gay NJ (1982) Distantly related sequences in the alpha- and beta-subunits of ATP synthase, myosin, kinases and other ATP-requiring enzymes and a common nucleotide binding fold. EMBO $J$ 1, 945-51.

Wyszynski FJ, Hesketh AR, Bibb MJ, and Davis BG (2010) Dissecting tunicamycin biosynthesis by genome mining: cloning and heterologous expression of a minimal gene cluster. Chem $S c i$ 1, 581-9. 